\title{
Component based Integration Testing using Abbot Tool
}

\author{
Neelam Sirohi \\ Research Scholar \\ H.C.T.M Kaithal
}

\author{
Anshu Parashar \\ Associate Professor \\ H.C.T.M Kaithal
}

\begin{abstract}
Testing is an important part in software development. When a component based software system are developed then to ensure the quality of system, testing must be done. Components have implementation transparency property and they are heterogeneous in nature. When the components are integrated to make a system then unexpected result may occur. So integration testing is necessary when components are integrated. In this paper, a student record system is implemented and its components are tested using abbot tool. Abbot tool is used for integration/functional testing. Abbot builds upon the java.awt.Robot class to provide an automated event generation and validation framework for Swing GUI components. The framework can be used to create, record, and execute scripts and programmatic test cases in Java. Abbot also has a script editor called Costello that facilitates the creation of scripts in XML. This paper also describes the features of abbot tool.
\end{abstract}

\section{General Terms}

Component based system, testing techniques.

\section{Keywords}

GUI Component Testing, XML Script, Abbot Tool, Costello Script Editor.

\section{INTRODUCTION}

Software testing is one of the most important part in software development process. Every programmer knows that testing is essential in the development of software application. Component based software systems are mainly constructed from reusable components such as third party components and Commercial off the Shelf Components (COTS). Due to these reusable and commercial off the shelf components, component based system are developed quickly, easily and with minimum resource cost [1]. Component-Based Development (CBD) offers a radically new approach to the design, construction, implementation and evolution of software applications. Software applications are implemented by assembling the several components; the components may be written in several different programming languages and run on several different platforms. Conventional development is a special case of CBD, which lacks some of the techniques and opportunities that characterize full CBD.

To ensure the best delivery of component based software, effective and efficient testing is the key process in software development. Component Based Software has implementation transparency property and they are written in several different programming languages and run on several different platforms. This heterogeneous nature of components raises difficulties in testing. In software development, several components are reused to build a system. Then the components must be tested adequately. Most of the testing tools are available today for GUI based testing like Marathon,
Guitar, Abbot, HTML Unit, HTML Fixture, Selenium. Marathon, Guitar and Abbot are java based tools where as HTML Unit, HTML Fixture, Selenium are web based tools. Junit tool is used for unit testing. Junit is a unit testing framework, which means that junit works by taking the smallest part of testable software, this tool isolate that part from rest of the software and in turn validating that it works correctly or not. Aim of this paper is component based integration testing by using testing tool - Abbot that is suitable for integration testing. It checks all the components together. Abbot framework is a java library that provides methods to reproduce user actions and examine the state of GUI components. The framework may be invoked directly from java code or accessed without programming through the use of scripts. Abbot tests the java UI. It provides a Costello script editor which is built on Abbot. Costello allows the tester to easily launch, explore, and control an application. Framework may be used with both scripts and compiled code. Costello script editor runs the $\mathrm{xml}$ scripts.

In this paper, student record system is implemented and all the components are tested. For testing these components abbot tool is used which provides an automated event generation and validation framework for GUI components. In this paper main purpose is to test the functionality of all the components that they are working properly after integrated.

The next section presents background information on the test generation. Section 3 describes the proposed work. Section 4 describes the implementation work done and results. Then section 5 conclusion and section 6 future work.

\section{RELATED WORK}

In the past, many papers have been published to address the component based testing and issues related to component based software engineering. Testing of component based system is different from normal software testing that's why the testing techniques are also bit different. There are basically two different approaches for testing white box and Black box. The black box testing is more prevalent in the component based systems because in most of the cases the source code is not available with the component; at most only the specification is available. Several techniques are defined to test the component based system. Some of them are defined below.

\subsection{Adequate testing}

An "Adequate Testing" approach for testing component based software was suggested by David S.Rosenblum [2] this technique provide initial basis for testing of component based software. The main result of this technique is the formal definition of the concept c-adequate-for $\mathrm{p}$ for adequate unit testing for the components and c-adequate -for $\mathrm{m}$ for integration testing for component based system. 


\subsection{Boundary value analysis}

Muthu Ramchandran[4] suggested "Boundary Value Analysis" for testing CBS. It is a software testing technique. In this technique test cases are designed in such a way that they include representatives of boundary values. It is based on complex embedded software and it also helps to apply boundary value testing and analysis on component interfaces which is the key to achieve testability of reusable software components. It uses visual notations to understand component connections/compositions.

\subsection{Integrated testing technique}

Sami Beydeda and Volkar gruhn et.al [3] proposed "Integration Testing Technique". In this all the features of component based system are tested and it allows more rigorous testing. The main purpose of this approach is to give graphical representation which combines the black and white box information. This graphical representation can then be used for test case generation. But this technique has one flaw that is very time consuming approach and there is no provision for automatic test case generation. This technique has one flaw that is very time consuming approach and there is no provision of automatic test case generation. Our next part shows the automatic test case generation testing techniques. After this automated testing techniques are proposed.

\subsection{Automated software robustness testing}

The trend shifted towards automatic testing of software components Marcel Dix Holger et al. [5] suggested "Automated Software Robustness testing". Main purpose of this technique is to do the automated robustness testing. Robustness is defined as the components ability to handle invalid input conditions. This technique can generate large number of test cases with small input values. But there is demand for reducing this large mass of test cases so another technique that is static testing was introduced for test case reduction. It reduces the number of test cases to be executed without affecting test accuracy and reliability but it was unable to reduce the numbers of test case and to generate test cases, human efficient expertise is needed.

\subsection{Self testing of component based system}

"Self Testing" was suggested by Fevzi Belli et al. [6]. It is widely accepted that conventional test methods are not necessarily adequate for testing of component based software. Also conventional test tools cause same problem for the automation of the test CBS, because the knowledge about the implementation of the CBS are essential to run the tests. But the component manufacturers are not willing to provide the component source code. This technique is based on black box testing and uses some features of capture/playback tools. It provides a framework for automation of user oriented component testing which reduces the test cost.

\subsection{Regression testing}

Chengiying Mao et.al[7] suggested "Regression Testing Technique". It is based on change information. Due to lack of information about source code of externally provided component system tester can't perform effective regression testing on their component based system. Component users don't know the details of change in components so they are not able to select the proper test cases to test modified components. This technique provides a regression testing method based on enhanced change information of component version to test the modified components. It needs a joint participation of component developer and the users. Call for graph is used to calculate the change information.

\subsection{Modular regression testing}

Bruce W.Weide [10] proposed "Modular Regression Testing". Whenever the modifications are done in the software system then regression testing must be done. On the release of every new version, new test cases are generated. It is too expensive to generate the new test cases for every version. Modular regression testing provides an advantage over regression testing. In this RTCM is used. RTCM reduces this cost by providing the concept of reusability. Regression Test Case Modeler (RTCM) is a framework that generates the test cases automatically from event forest and also generates regression test suite. RTCM choose old test suite which represent correct input and is necessary to validate the modified software. When the structure of the original GUI is modified, test cases from the original GUI are either usable or unusable on the modified GUI. Test cases which can't be used in modified GUI are discarded and test cases which can be used in modified GUI are kept. Test cases which can't be rerun are known as obsolete test cases. GUI test cases can be unusable, usable or repairable. In RTCM we have followed the principle "do not throw away unusable test cases".

\subsection{Object oriented component testing}

Fakhra Jabeen et.al[8] suggested "Object Oriented Testing". The unavailability of source code preludes extrapolating standard testing approaches. In this technique object oriented framework is proposed that relies on utilization of discrete descriptors to facilitate test execution and to enable a uniform information flow. At present it supports component unit testing and partial integration testing.

Junit tool is used for unit testing. Abbot tool is a most popular tool and used for integration/functional testing.

Its features include:

- Abbot is an extension of junit tool and is used for integration/ functional testing.

- Provides GUI tests for java AWT/swing application.

- Consists of recorder, player and an editor.

- Records tests script in java.

- Allows to write test cases directly from java code.

- Allows to insert assertions in script easily.

- It runs the XML scripts then there is no need of compilation.

\section{PROPOSED WORK}

Software testing is a critical element of any kind of software's quality assurance and is of utmost important for the credibility of the software system. When it comes to component based software paradigm, testing mechanism and strategies varies from the traditional testing approaches because traditional software system contained source code which can be tested by tester, but while testing component based software system the source code is not available generally. Testing is especially important for system being assembled from any self contained software components. GUI's by far are the most important means, of communicating with today's software, so the functional characteristics of GUI's are of utmost important. The test data required for testing GUI is huge sometime so manual approach isn't suitable. Manual testing makes regression task more laborious and time consuming and 
longer times are needed for test coverage. Automatic testing of software is necessary to save the time and to reduce the labour of programmers. In software development unit testing is the starting phase of testing. First of all unit testing is done. In unit testing a single piece of software/single component is tested by the tester. Junit tool is used for automatic unit testing. This tool works on java platform. Integration testing comes after unit testing and is most important testing in software development. Several components are integrated to make a system. Testing of independent component is simple but when the components are assembled in a system then unexpected results may occur. Then to ensure the quality of the system, integrated testing is necessary. Abbot tool is used for integration testing and it is an extension of junit tool or can be says that a better bot.

The ultimate goal of this paper is to completely automate the testing of integrated components. A programmer should be able to perform testing simply by clicking on a button. Abbot tool provides GUI integration/functional testing and test cases are generated automatically by this tool. In this paper, student record system is implemented and all the components are tested together. Next section describes the implementation and results of the work.

\section{IMPLEMENTATION AND RESULTS}

Abbot tool provides a framework to test the GUI components. Abbot builds upon the java.awt.Robot class. It provides an automated event generation and validation framework for Swing GUI components. The framework can be used to create, record, and execute scripts and programmatic test cases in Java. Abbot also has a script editor called Costello that facilitates the creation of scripts in XML. This tool converts the java source code into .jar (java archive files) files which are executable files and path of these files are associated with $\mathrm{xml}$ files and give them to abbot then abbot automatically generates test cases. A student record program is implemented in java. After this xml scripts are created and these scripts are used by Costello script editor to run the applications. To test this program open the Costello (script editor), then LaunchMain.main([]) method occur. With the help of this method tester can run XML script. This method is shown in the Fig.1. This fig also shows the hierarchy, refrences, properties, attributes.etc. If the hierarchy of any component is changed then the script will break. This will produce an error in the system. Then to remove this type of error, script must be updated after every change in the hierarchy of components.

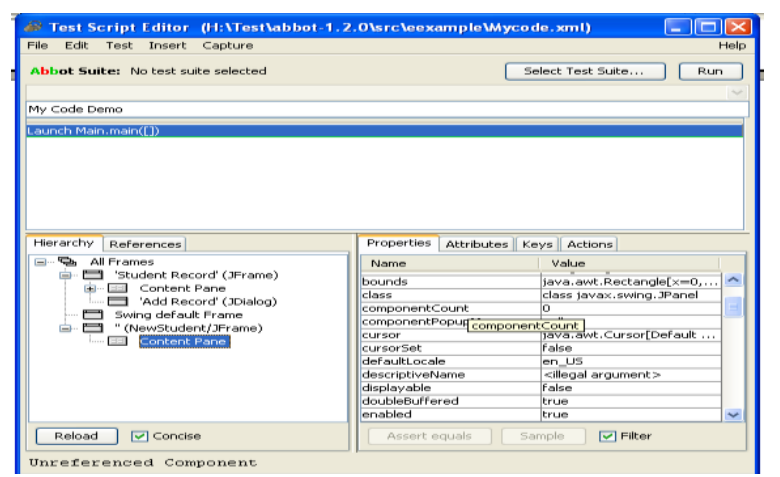

Fig.1 Costello script editor. It shows LaunchMain.main([]) method which run xml script of student record.
Example of abbot test code for student record is shown below. This java code is used for inserting information about student.

import java.awt.*;

import java.awt.event.*;

import javax.swing.*;

public class Add extends AbstractAction

\{

private JDialog dialog;
private JFrame frame;

public Add(String commandAction,JFrame frame) \{

super(commandAction);

this.frame = frame;

\}

public void actionPerformed(ActionEvent event)

\{

addNewStudent();

\}

public void addNewStudent()

Record",true);

dialog = new JDialog(frame,"Add

dialog.setContentPane(new

NewStudent(dialog).getPanel());

dialog.pack();

dialog.setLocationRelativeTo(frame);

dialog.setVisible(true);

\}

\}

After click on run button student record window open in front of tester then tester can check all the validation check which put on every component for testing. If all the test cases are passed without any error then result is shown in bottom of costello like invoking test case.. done as shown in fig 2 . Tester also able to tell what test failed because it is highlighted in red line and error message appears in bottom of Costello. In this, all the textboxes, dropdownlist and command buttons i.e add, close are tested that they are working correctly or not.

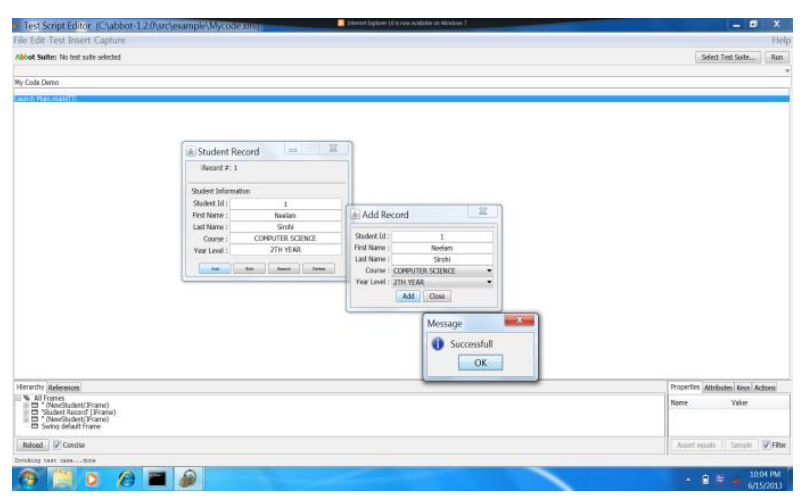

Fig.2 Testing of addition component using abbot tool.

Similarly delete, search and edit components are tested by this tool. This is an example of student deletion. 
import java.awt.*;

import java.awt.event.*;

import javax.swing.*;

public class Delete extends AbstractAction

Private StudentList studentList $=$ new StudentList () private JFrame frame $=$ null

public Delete(String commandAction,JFrame

frame)

\{

super(commandAction);

this.frame = frame;

public void actionPerformed(ActionEvent event)

$$
\text { if(studentList.getList().size() !=0) }
$$

Int

$=$

JOptionPane.showConfirmDialog(frame,"Are you sure? you want to delete this record 'student'","Confirm delete",JOptionPane.YES_NO_OPTION);

$$
\begin{aligned}
& \text { if }(\mathrm{n}==\text { JOptionPane.YES_OPTION }) \\
& \text { \} } \\
& \text { deleteStudentRecord(); } \\
& \} \\
& \text { public void deleteStudentRecord() } \\
& \text { \{ }
\end{aligned}
$$

studentList.getList().remove(studentList.getRecord(

) )

0)

$$
\text { if(studentList.getList().size() != }
$$$$
\{
$$$$
\text { Student student }
$$

(Student)studentList.getList().getLast();

1);

$$
\text { studentList.setRecord(studentList.getList().size() - }
$$

new StudentRecordGUI(studentList.getRecord ()$+1)$;

$$
\text { new }
$$

StudentRecordGUI(student.getFirstName(),

$$
\text { student.getLastName(), }
$$$$
\text { student.getIdNum(), }
$$$$
\text { student.getCourse(), }
$$$$
\text { student.getYearLevel()); }
$$$$
\text { \} }
$$$$
\text { else }
$$$$
\{
$$

new StudentRecordGUI(false); new StudentRecordGUI(0); new StudentRecordGUI("","",0,"","");
After click on delete button of application a message box appears in front of tester into the figure "Are you sure? you want to delete this record 'student' ". Two command buttons appears, yes or no. This is shown in fig 3 .

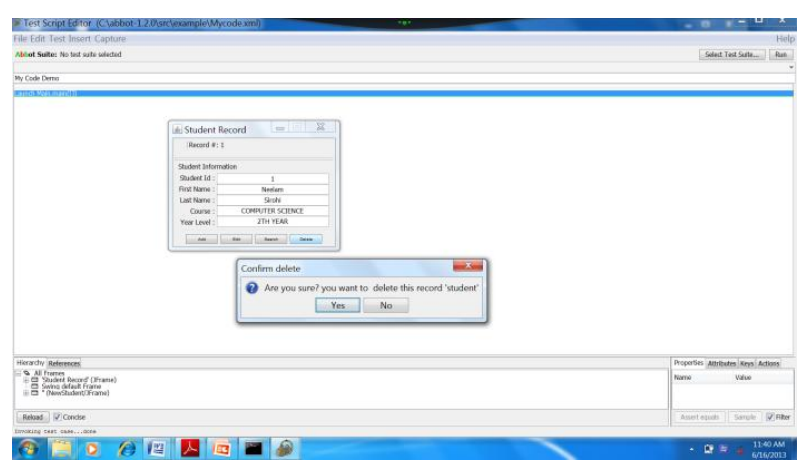

Fig.3 Testing of delete component of student record.

Searching component depends upon the addition component. It can search only those students information whose records are added i.e depend on the addition component.

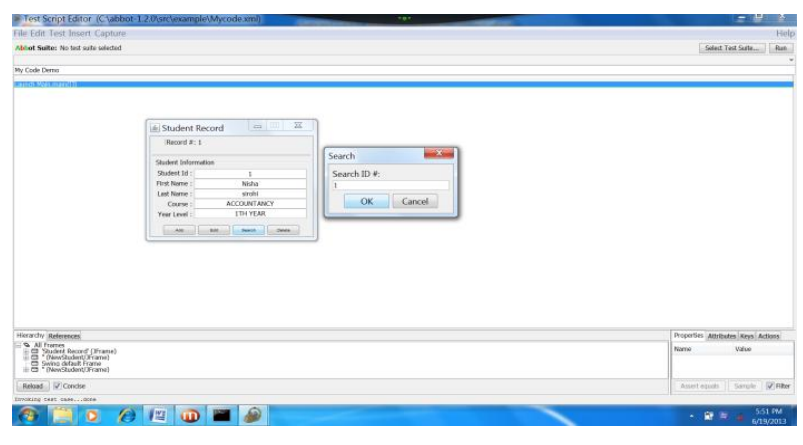

Fig.4 Testing of search component of student record

From the above results it is sure that student record application works correctly. All test of components are passed by abbot tool.

\section{CONCLUSION}

This paper presents a systematic integration testing using abbot tool. Abbot tool provides integration/functional testing and it is an extension of junit. It helps writing system/GUI test for java AWT/swing application. Abbot tool automatically generates the test cases. We also define the role of Costello script editor. Costello allows us to easily launch, explore, and control an application. The framework may be used with both scripts and compiled code. Costello script editor can also run $\mathrm{xml}$ scripts and results are shown in the bottom.

\section{FUTURE WORK}

This paper discusses the features of abbot testing tool. Student record application is implemented in java and observes that how abbot testing tool can help in testing the components of this application. This paper presents the testing of static components. Dynamic objects are another thing which keeps on changing like we see on some of the website, text logo's keeps on changing in the same area. In future some work can be done on dynamic components. 


\section{REFERENCE}

[1] Jim Q Ning, 1997. Component-Based Software Engineering, Proceedings of U.S National Institute of Standards and Technology's Advance Technology Program on Component Based Software, Document number 0-8186-7940-9/97.

[2] David S. Rosenblum, 11 Aug 1997. Adequate Testing of Component-Based Software, Technical Report 97-34 Department of Information and Computer Science University of California, Irvine, CA 92697-3425.

[3] Sami Beydeda and Volker Gruhn, IEEE Computer Society 2001. An Integrated testing Technique for Component-Based Software.

[4] Muthu Ramchandran, 2003. Testing Software Components using Boundary Value Analysis, proceedings of the $29^{\text {th }}$ EUROMICRO conference New Waves in System Architecture" (EUROMICRO 08), Document number 1089-6503/03, IEEE Computer Society.

[5] Marcel Dix. Holger and D. Hofmann, 2002. Automated Software Robustness Testing, proceedings of the 28th Conference (EUROMICRO'02) IEEE, Document number 1089-6503/02.
[6] Fevzi Belli and Christof J. Budnik, 2005. Towards SelfTesting of Component-Based Software, Proceeding of the $29^{\text {th }}$ Annual International Software and Applications Conference (COMPSAC '05), IEEE Computer Society.

[7] Chengying Mao, Yansheng Lu, 2005. Regression Testing for Component-based Software Systems by Enhancing Change Information, Proceedings of the Asia-pacific Software Engineering Conference (APSEC'05), Document number 0-7695-2465-6/05.

[8] Fakhra Jabeen, Muhammad jaffar-Ur Rehman, 2005. A Framework for Object oriented Component Testing, Document number 0730-3157/05, IEEE press.

[9] Nitin V. Koppalkar, Seshaiah Uppala and Mahesh Madugundu. Testing of Component-Based Software Systems, Philips Research India-Bangalore.

[10] Bruce W.Weide, 2005. Modular Regression Testing, Connections to Component-Based Software, Dept. of Computer and Information Science, The Ohio State University , 2015 eil Ave. Columbus, OH 43210,USA +1614292 .

[11] A. M.Memon and M. L.Soffa. Regression testing of GUIs , in Proceedings of the $9^{\text {th }}$ European Software Engineering Conference (ESEC) and 11th ACM SIGSOFT International Symposium on the Foundations of Software Engineering 